ARTICLE

Received 15 Jan 2015 | Accepted 22 May 2015 | Published 26 Jun 2015

DOI: $10.1038 /$ ncomms $8598 \quad$ OPEN

\title{
Extreme ultraviolet imaging of three-dimensional magnetic reconnection in a solar eruption
}

J.Q. Sun ${ }^{1}$, X. Cheng ${ }^{1}$, M.D. Ding ${ }^{1}$, Y. Guo ${ }^{1}$, E.R. Priest ${ }^{2}$, C.E. Parnell ${ }^{2}$, S.J. Edwards ${ }^{3}$, J. Zhang ${ }^{4}$, P.F. Chen ${ }^{1} \&$ C. Fang ${ }^{1}$

Magnetic reconnection, a change of magnetic field connectivity, is a fundamental physical process in which magnetic energy is released explosively, and it is responsible for various eruptive phenomena in the universe. However, this process is difficult to observe directly. Here, the magnetic topology associated with a solar reconnection event is studied in three dimensions using the combined perspectives of two spacecraft. The sequence of extreme ultraviolet images clearly shows that two groups of oppositely directed and non-coplanar magnetic loops gradually approach each other, forming a separator or quasi-separator and then reconnecting. The plasma near the reconnection site is subsequently heated from $\sim 1$ to $\geq 5$ MK. Shortly afterwards, warm flare loops ( $\sim 3 \mathrm{MK}$ ) appear underneath the hot plasma. Other observational signatures of reconnection, including plasma inflows and downflows, are unambiguously revealed and quantitatively measured. These observations provide direct evidence of magnetic reconnection in a three-dimensional configuration and reveal its origin.

\footnotetext{
${ }^{1}$ School of Astronomy and Space Science, Nanjing University, Nanjing 210093, China. ${ }^{2}$ School of Mathematics and Statistics, University of St Andrews, Fife, KY16 9SS Scotland, UK. ${ }^{3}$ Department of Mathematical Sciences, Durham University, Durham DH1 3LE, UK. ${ }^{4}$ School of Physics, Astronomy and Computational Sciences, George Mason University, Fairfax, Virginia 22030, USA. Correspondence and requests for materials should be addressed to X.C. (email: xincheng@nju.edu.cn) or to M.D.D. (email: dmd@nju.edu.cn).
} 
$\mathrm{M}$ agnetic reconnection plays an important role in various astrophysical, space and laboratory environments ${ }^{1}$ such as $\gamma$-ray bursts ${ }^{2}$, accretion disks ${ }^{3,4}$, solar and stellar corona $^{5,6}$, planetary magnetospheres ${ }^{7,8}$ and plasma fusion ${ }^{9,10}$. In the classic two-dimensional (2D) model, reconnection occurs at an X-point where anti-parallel magnetic field lines converge and interact. As a consequence, free energy stored in the magnetic field is rapidly released and converted into other forms of energy, resulting in heating and bulk motions of plasma and acceleration of non-thermal particles ${ }^{11}$. In the past decades, much attention has been paid to validate this picture. One piece of direct evidence is from in situ solar wind measurements at the magnetosheath and magnetotail of the Earth ${ }^{12}$. Most observational evidence is from remote sensing observations of solar flares, including cuspshaped flare loops ${ }^{13}$, plasma inflows/outflows ${ }^{14,15}$, downflows above flare arcades ${ }^{16}$, double hard X-ray coronal sources ${ }^{17}$, current sheets ${ }^{18}$ and changes in connectivity of two sets of EUV loops during a compact flare ${ }^{19,20}$. With many of these observations, researchers were trying to reveal the $2 \mathrm{D}$ aspects of reconnection. However, reconnection is in reality a process in 3D that occurs in places where magnetic connectivity changes significantly, namely, at null points ${ }^{21}$, separators ${ }^{22,23}$ or quasiseparators ${ }^{24}$.

Recently launched spacecraft Solar Terrestrial Relations Observatory (STEREO) and Solar Dynamics Observatory (SDO) provide us an unprecedented opportunity to observe reconnection in a 3D setting. Utilizing stereoscopic observations from these two spacecraft, 3D configurations of various solar phenomena have been reconstructed ${ }^{25,26}$. Here we study reconnection through its reconstructed 3D magnetic topology as well as many other signatures. The Extreme Ultraviolet Imager $(\mathrm{EUVI})^{27}$ on board STEREO and the Atmospheric Imaging Assembly (AIA) ${ }^{28}$ on board SDO provide the necessary observational data; in particular, the AIA has an unprecedented high spatial resolution ( 0.6 arcsec per pixel), high cadence (12 s) and multi-temperature imaging ability (10 passbands).

\section{Results}

Overview of the reconnection event. The event of interest occurred on 27 January 2012, when STEREO-A and SDO were separated in space by 108 degrees along their ecliptic orbits (Fig. 1a). From $\sim 00: 00$ to 03:00 UT (universal time), a pre-existing large-scale cavity, which refers to the dark region in the EUV or soft X-ray passbands and is usually interpreted to be the cross-section of a helical magnetic flux rope ${ }^{29-31}$, appears above the western solar limb as seen from the Earth. The reason why the cavity is dark may be that the density has decreased or that the plasma temperature has increased to a value outside the effective response of the lower temperature passbands. The cavity, mostly visible in the AIA $171 \AA$ passband (sensitive to a plasma temperature of $\sim 0.6 \mathrm{MK}$ ), starts to expand and rise from $\sim 01: 40$ UT, and finally results in a coronal mass ejection (CME) that is well observed by the AIA and the Large Angle and Spectrometric Coronagraph (LASCO $)^{32}$ on board Solar and Heliospheric Observatory (SOHO; Fig. 1b). The slow rise of the cavity causes its two legs, which are made of cool loops, to approach each other and form an X-shaped structure near 03:00 UT (Fig. 1c,d and
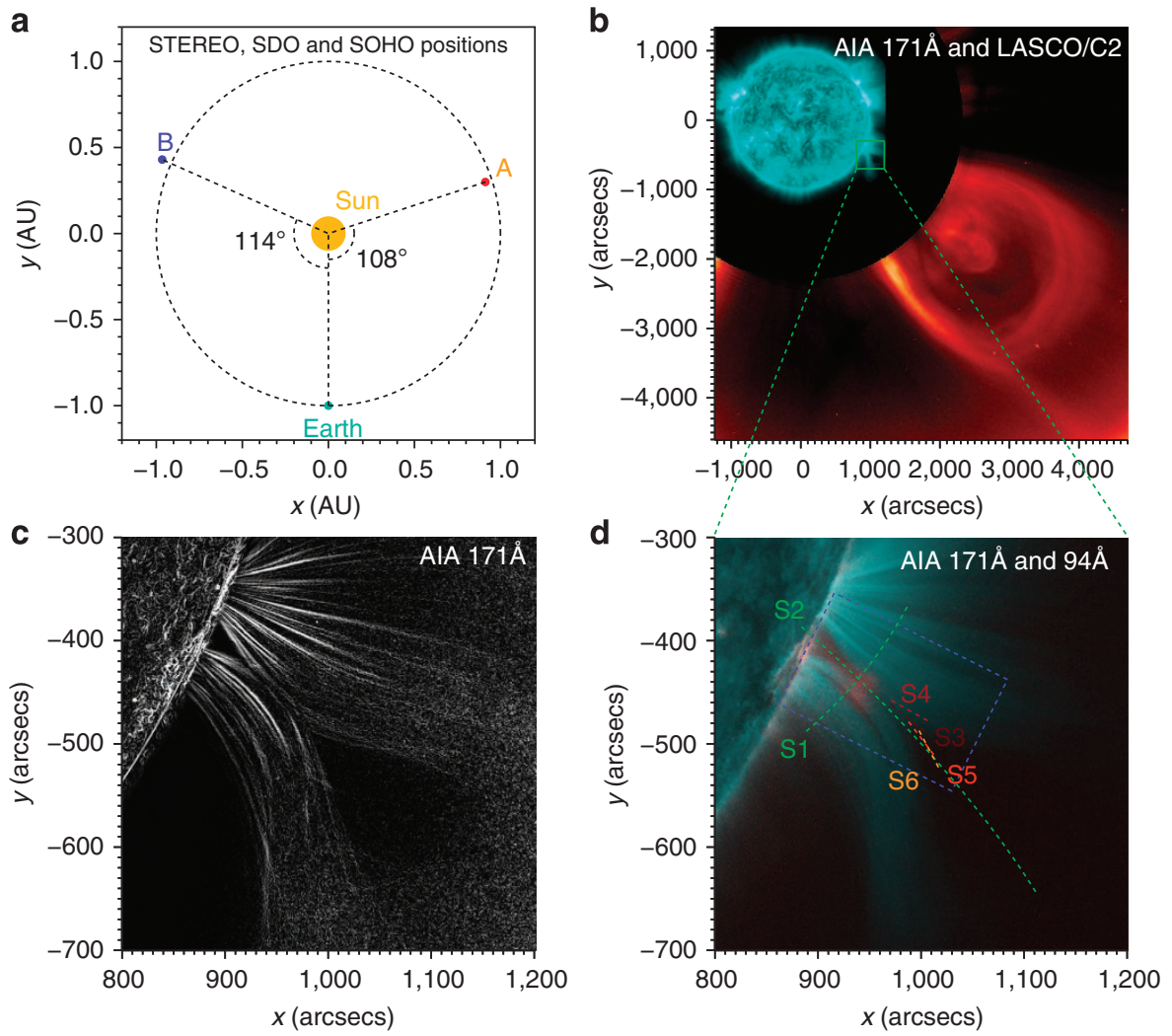

Figure 1 | Overview of the 17 January 2012 solar flare and CME reconnection event. (a) The positions of the Sun, Earth and STEREO-A/B satellites ( $\mathrm{SOHO}$ is at L1 point and SDO is in the Earth orbit). (b) A composition of the AIA $171 \AA$ passband image (cyan) and the LASCO C2 white-light image (red). The green box indicates the main flare region. (c) The enhanced AIA $171 \AA$ image showing a clear X-shaped structure. (d) A composite image of the AIA $171 \AA$ (cyan) and $94 \AA$ (red) passbands. Cyan (red) indicates coronal loops with a temperature of $\sim 0.6 \mathrm{MK}(\sim 7.0 \mathrm{MK})$. Six dashed lines denote six slices (S1-S6) that are used to trace the evolution of various reconnection features with time. 
Supplementary Movies 1 and 2). Following the disappearance of the cool loops (cyan in Fig. 1d), a hot region ( $\sim 7 \mathrm{MK}$; visible in the AIA $94 \AA$ passband) immediately appears near the X-shaped structure, indicating the initial heating of a solar flare (red in Fig. 1d). Unfortunately, as the flare soft X-ray emission is very weak and submerged in the emission from the decay phase of a previous flare, the accurate magnitude of the flare is not recorded by Geostationary Operational Environmental Satellite. We also note that there are no X-ray observations from the Reuven Ramaty High Energy Solar Spectroscopic Imager because of annealing.

3D topology and origin of magnetic reconnection. Observations from SDO (the AIA $171 \AA$ passband; Fig. 2a), in combination with STEREO-A observations (the EUVI $171 \AA$ passband; Fig. 2b), enable us to reconstruct the $3 \mathrm{D}$ topology of the reconnection and its evolution. Owing to the high magnetic Reynolds number of the ionized corona, the plasma is frozen to the magnetic field; and so the loop-like plasma emission is reasonably assumed to outline the geometry of the magnetic field ${ }^{33}$. We select two magnetic loops (cyan and green dashed lines in Fig. 2a,b) that can most clearly exhibit the reconnection process. With images from two perspective angles, the $3 \mathrm{D}$ structure of the loops is reconstructed (Fig. 2c and Supplementary Movie 3). The results display a clear picture of how the connectivity of the loops changes as the reconnection proceeds. Before reconnection, two nearly oppositely directed loops are anchored, respectively, at each side of the filament in the active region (left panel of Fig. 2b). The plasma between their legs has been heated to a moderate temperature (left panel of Fig. 2c).

With the rise of the cavity, the underlying loops of opposite polarities gradually approach each other. As the inward movements of the loops are not coplanar, an apparent separator or quasi-separator appears at 04:14 UT (middle panel of Fig. 2b). We calculate the 3D global magnetic field on January 26 using the potential field assumption ${ }^{34}$ and find an absence of pre-existing null points and separators in the reconnection region. However,
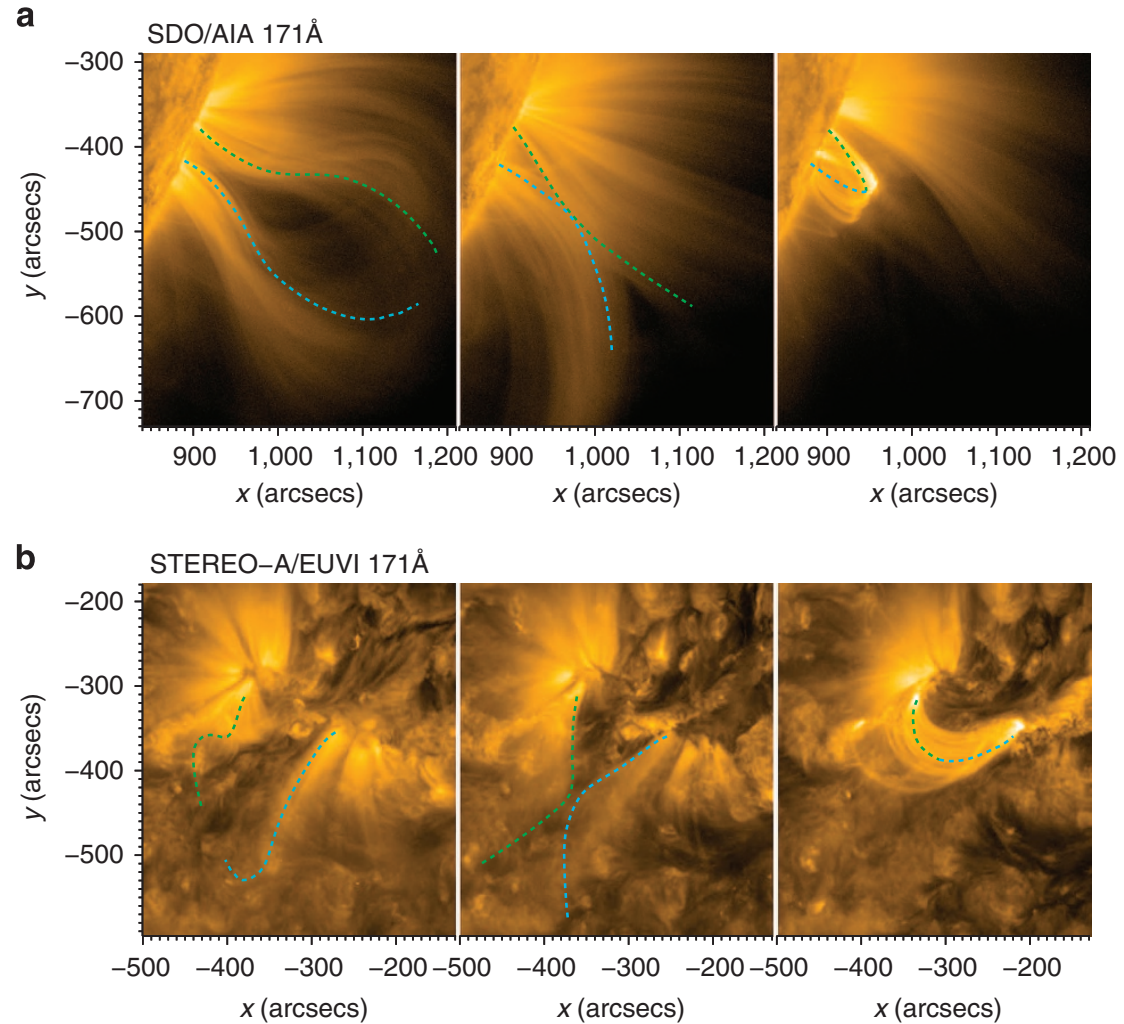

c

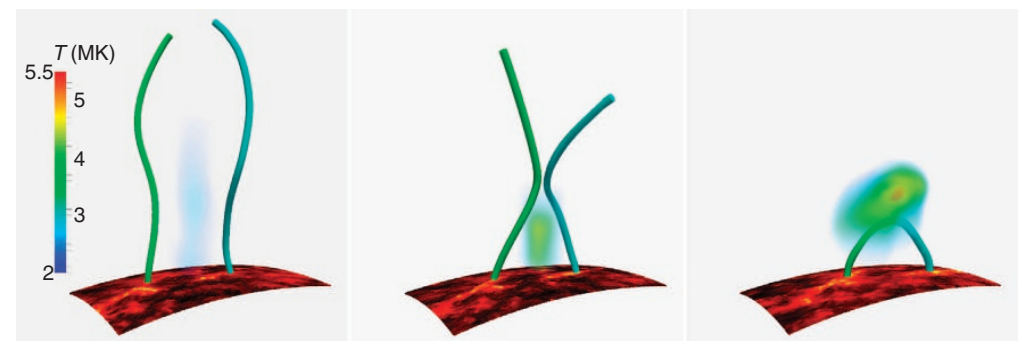

Figure 2 | Plasma and magnetic configurations during the reconnection process. (a) The AIA 171 $\AA$ images at 02:14 UT (left), 04:14 UT (middle) and 08:14 UT (right) displaying the side view of the evolution of two sets of coronal loops. The cyan and green dashed curves show selected coronal loops representing two magnetic field lines involved in the process. (b) The EUVI $171 \AA$ images showing the top view of the reconnection. The cyan and green dashed curves give another view of the same loops as in panel a. (c) The reconstructed 3D magnetic topology (cyan and green curves) and heated regions (cloud-like structures) before, during and after the reconnection. The bottom boundaries are the projected EUVI $304 \AA$ images showing the footpoints of the flare and the separation of two flare ribbons. 
the simple magnetic field in the original bipolar source region is strongly sheared from January 21 as shown by the long-existing filament/prominence at the bottom of the cavity (Fig. 2b). It suggests that a new separator or quasi-separator is formed with the prominence taking off (middle panel of Fig. 2c). As the reconnection initiates, free magnetic energy starts to be released, the most obvious consequence of which is to form a hotter region underneath the reconnection site.

Topologically, the reconnection between the two groups of loops forms poloidal field lines above the reconnection site, increasing the twist of the erupted flux rope. At the same time, a cusp-shaped field below the reconnection site quickly shrinks into a semicircular shape to form flare loops ${ }^{35}$ (right panel of Fig. 2c). With the acceleration of the CME, more plasma is heated to temperatures up to $\sim 5 \mathrm{MK}$, suggesting an enhanced reconnection. However, the heated region is still confined between the reconnection site and the flare loop top but with a spatial extension.

Quantitative properties of magnetic reconnection. AIA observations with high spatial and temporal resolution successfully capture evidence for reconnection including bilateral inflows, instantaneous heating of plasma near the reconnection site, and downflows that are related to the reconnection outflows ${ }^{16}$. To quantitatively investigate the inflows, we select an oblique slice in the 171 and $94 \AA$ composite images (S1 in Fig. 1d). The time-distance plot (Fig. 3a) clearly shows that the bilateral cool loops (cyan) keep converging to the middle (reconnection) region from $\sim 00: 00 \mathrm{UT}$. Once the visible innermost loops come into contact at $\sim 03: 10 \mathrm{UT}$, they immediately disappear; meanwhile, hot plasma (red) appears at the reconnection site. Several trajectories for inflows are tracked. The velocities of the inflows vary from 0.1 to $3.7 \mathrm{~km} \mathrm{~s}^{-1}$ (Fig. 3b). Moreover, for each trajectory, the velocity tends to increase towards the reconnection site, indicating that an inward force exists on both sides of the current sheet to accelerate the inflows.

The time-distance plot for the vertical slice (S2) shows the eruption of the CME and the downflows above the flare loops (Fig. 3c). In the early phase, only the slow rise of the CME cavity is detectable. However, along with the fast eruption of the CME, magnetic reconnection is initiated, which causes the plasma at the bottom to be rapidly heated. During this process, many dark voids intermittently appear above the heated region, rapidly falling, propagating a distance of $\sim 20-30 \mathrm{Mm}$, and finally disappearing. The time-distance plots (Fig. 3d) for four selected slices (S3-S6) show that the different downflows have almost similar trajectories. Their velocities range from $\sim 100$ to $200 \mathrm{~km} \mathrm{~s}^{-1}$ initially, but quickly decrease to tens of $\mathrm{km} \mathrm{s}^{-1}$ (Fig. 3e).

Role of magnetic reconnection in the flare and CME. The 2D temperature maps (Fig. 4a) and the time-distance plot of the temperature along the vertical slice (Fig. 4b) reveal the detailed temperature evolution of the heated region and flare loops. Before
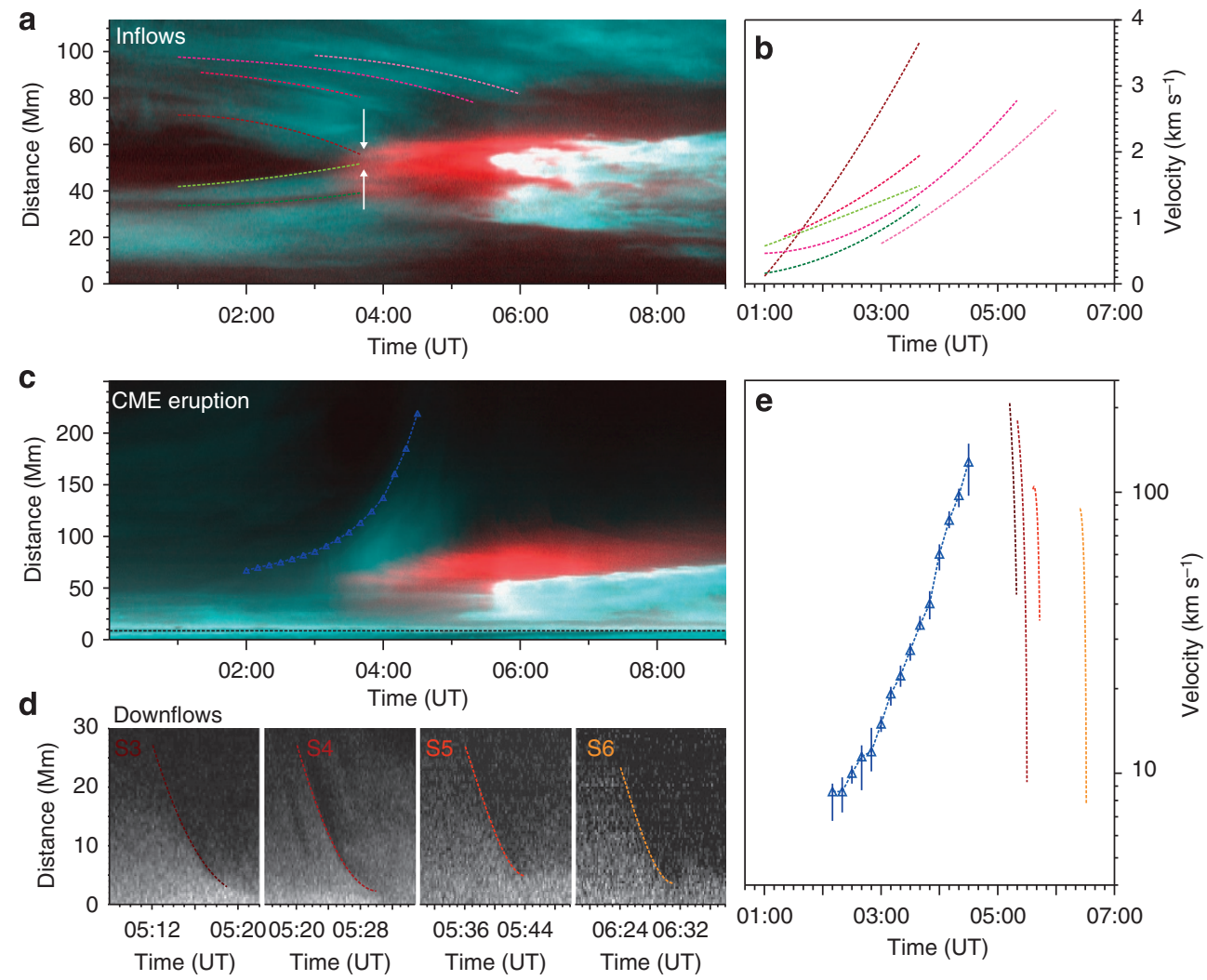

Figure 3 | Temporal evolution of plasma inflows and downflows during the reconnection. (a) A time-distance plot of the composite AIA $171 \AA$ (cyan) and $94 \AA$ (red) images along the direction of the inflows (denoted by S1 in Fig. 1d) showing the approach of oppositely directed loops (two white arrows). The dashed lines with different colours (green to pink) denote the height-time measurements of the inflow at different locations. (b) The velocities of inflows, which are derived by cubic-fitting to the height-time data. (c) A time-distance plot of the composite AIA $171 \AA$ (cyan) and $94 \AA$ (red) images along the rising direction of the CME (denoted by S2 in Fig. 1d). The blue dashed line denotes the height-time measurement of the CME bubble.

(d) The time-distance plots of the AIA $94 \AA$ images along the direction of four selected downflows (S3-S6 in Fig. 1d). The dashed lines are the height-time measurements of the downflows. (e) The velocities of the CME (blue) and four downflows (brown to yellow). The error in the velocity of the CME (marked by the vertical symbol size) mainly comes from the uncertainty of the height, which is taken as the s.d. of 10 measurements. 

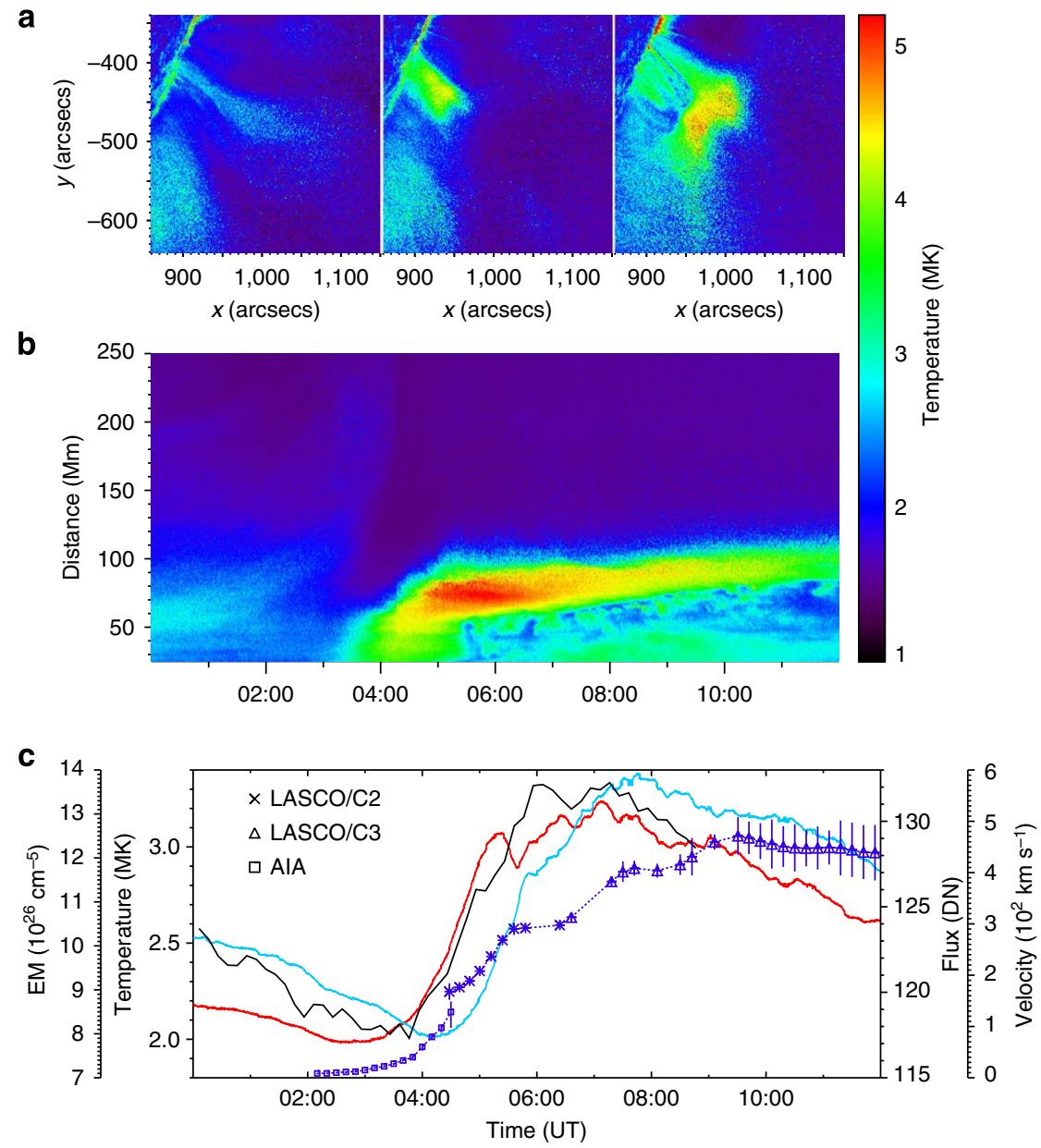

Figure 4 | Temporal evolution of the flare heating and the CME acceleration. (a) The DEM-weighted temperature map at three instants (02:14, 04:14 and 08:14 UT) showing the location of the region heated by the reconnection. (b) A time-distance plot of the temperature map along the rising direction of the CME (denoted by S2 in Fig. 1d) illustrating the temperature evolution of the CME bubble and the flare region. (c) The temporal evolution of the CME velocity (blue), the flare emission intensity in the EUVI $304 \AA$ passband (black, a proxy of the flare soft X-ray flux), mean temperature (red) and total EM (cyan). The error in the velocity (marked by the vertical symbol size) mainly comes from the uncertainty of the height, which is taken as the s.d. of 10 measurements.

the onset of the flare, the CME cavity is actually hotter than the surrounding coronal plasma, supporting the recent argument that it is most likely a pre-existing hot magnetic flux rope ${ }^{30,31}$. Owing to the rise motion of the hot cavity, the plasma underneath the reconnection region is quickly heated, forming a hot region with an average temperature of $\sim 4 \mathrm{MK}$. In the hot region, some flare loops are discernible. As the reconnection continues, the hot region further ascends and extends, and its temperature rises to $>5 \mathrm{MK}$. In contrast, the originally formed hot flare loops gradually cool down to $\sim 2 \mathrm{MK}$ and become more distinctive in the temperature maps. Meanwhile, newer hot flare loops are formed, stacked over the cool ones. The above process continues until the reconnection stops.

We deduce in detail the evolution of the emission intensity, DEM-weighted temperature, total emission measure (EM) of the flare region and the velocity of the CME (Fig. 4c). The CME velocity is mostly synchronous with the intensity increase and plasma heating of the flare but precedes the total EM by tens of minutes. From $\sim$ 04:00 to 05:30 UT, the CME velocity, the flare intensity and the temperature rapidly increase. From $\sim 05: 30$ to 08:00 UT, the CME is still being accelerated, but the intensity and temperature of the flare reach a maximum with only some minor fluctuations. Afterwards, the intensity and temperature start to decrease; the CME velocity also decreases to a nearly constant value, most likely as a result of the interaction with the background solar wind ${ }^{36}$. On the basis of the velocity of the inflows and downflows, a lower limit on the reconnection rate (the inflow Alfvén Mach number) is estimated to be 0.001-0.03, which is able to produce the observed weak solar flare and the long-accelerating slow CME.

\section{Discussion}

We have reconstructed the 3D magnetic topology of the fast reconnection in a solar eruption and quantify the properties of the reconnection and its role in the flare and CME. The method of direct imaging overcomes the disadvantage of magnetic field extrapolations based on non-linear force-free field modelling when studying a dynamic process ${ }^{37}$. The excellent observations provide much needed elucidation of the physical processes involved in a flare/CME in a $3 \mathrm{D}$ configuration. In a traditional $2 \mathrm{D}$ flare model $^{6}$, the initial magnetic configuration consists of two sets of oppositely directed field lines. A current sheet formed between them is an essential ingredient for flare occurrence. Once the reconnection begins, magnetic energy is released to produce an enhanced flare emission, and post-flare loops are formed, 
mapping to two flare ribbons on the chromosphere. The discovery of a pre-existing flux rope makes a crucial addition to the standard paradigm ${ }^{31}$. It suggests that the reconnection is associated with the eruption of the flux rope. Although the flux rope is a $3 \mathrm{D}$ structure, observations made thus far of the reconnection are mostly restricted to $2 \mathrm{D}$, in which the flux rope often appears as a hot plasma blob when viewed along the axis, and the reconnection site underneath the blob is apparently manifested as a thin and long sheet ${ }^{30}$. In a $3 \mathrm{D}$ case, the magnetic topology becomes much more complex and there are different regimes of magnetic reconnection ${ }^{38}$. The event studied here does reveal some new features. First, the reconnection site is more likely a separator or quasi-separator. The fact that the two sets of loops that are obviously non-coplanar are approaching each other does imply the presence of a separator or quasi-separator between them. Second, in the 2D case, reconnection forms an isolated closed field (a section of a flux rope) above, in addition to a flare loop below; while in the $3 \mathrm{D}$ case, reconnection supplies poloidal flux to the flux rope whose two ends are still anchored on the solar photosphere. As the reconnection proceeds, more and more poloidal flux is added to the flux rope, further accelerating the $\mathrm{CME}$ and in turn strengthening the flare emission. Our results are consistent with and lend observational support to models of flux rope-induced solar eruptions ${ }^{39}$. The primary trigger of the eruption may be torus or kink instability of a pre-existing flux rope ${ }^{40}$, while magnetic reconnection, which occurs at a newly formed separator ${ }^{22,23}$ or quasi-separator ${ }^{24}$, releases free magnetic energy and helps accelerate the eruption.

\section{Methods}

3D reconstruction and visualization. Using the Interactive Data Language (IDL) program 'scc_measure.pro' in the Solar SoftWare (SSW) package, we reconstruct the $3 \mathrm{D}$ coordinates of the magnetic loops. This routine allows us to select a point in, for example, the AIA image. A line representing the line-of-sight from the AIA perspective is then displayed in the image from other perspectives, such as EUVI. According to the emission characteristics, we identify the same point at this line. $3 \mathrm{D}$ coordinates of the selected point (heliographic longitude, latitude and radial distance in solar radii) are then determined. With the same manipulation, the $3 \mathrm{D}$ coordinates of all the points along the magnetic loops are derived. For each loop, the reconstruction is repeated 10 times, the most optimal one of which is chosen as the result, thus ensuring the accuracy of the reconstruction. To trace the evolution of the magnetic loops, we keep their footpoints fixed. The $3 \mathrm{D}$ visualization is realized by the software Paraview.

Differential emission measure reconstruction. The differential emission measure (DEM) is recovered from six AIA passbands including $94 \AA$ (Fe X, $\sim 1.1 \mathrm{MK}$; Fe XVIII, $\sim 7.1 \mathrm{MK}), 131 \AA$ (Fe VIII, $\sim 0.4 \mathrm{MK}$; Fe XXI, $\sim 11 \mathrm{MK}$ ), $171 \AA$ (Fe IX, $\sim 0.6 \mathrm{MK}), 193 \AA$ (Fe XII, $\sim 1.6 \mathrm{MK}), 211 \AA$ (Fe XIV, $\sim 2.0 \mathrm{MK}$ ) and $335 \AA$ (Fe XVI, $\sim 2.5 \mathrm{MK})$ through the regularized inversion method ${ }^{41}$. The observed flux $F_{i}$ for each passband can be written as:

$$
F_{i}=\int \operatorname{DEM}(T) R_{i}(T) \mathrm{d} T+\delta F_{i},
$$

where $R_{i}(T)$ is the temperature response function of passband $i, \operatorname{DEM}(T)$ indicates the plasma DEM in the corona, and $\delta F_{i}$ is the error of the observational intensity for passband $i$. The temperature range in the inversion is chosen as $5.5 \leq \log T$ $\leq 7.5$. With the derived DEM, the DEM-weighted (mean) temperature and the total EM are calculated as:

$$
T_{\text {mean }}=\frac{\int_{T_{\min }}^{T \max } \operatorname{DEM}(T) T \mathrm{~d} T}{\int_{T \min }^{T \max } \operatorname{DEM}(T) \mathrm{d} T}
$$

and

$$
E M=\int_{T \min }^{T \max } \operatorname{DEM}(T) d T .
$$

The temperature range of integration is set to be $5.7 \leq \log T \leq 7.1$, within which the EM solutions are well constrained as shown in Supplementary Fig. 1. Finally, with the mean temperature at each pixel, the $2 \mathrm{D}$ temperature maps are constructed.

Enhancement of EUV images. To display the fine structures of the EUV images, we enhance the contrast by the routine 'aia_rfilter.pro' in SSW. This program first sums five images and divides the summed image into a number of rings. Each ring is then scaled to the difference of the maximum brightness and the minimum one. The final images are obtained by performing the Sobel edge enhancement taking advantage of the IDL program 'sobel.pro'.

3D magnetic field extrapolation and singularity calculation. With a potential field model, we extrapolate the 3D global magnetic field structure using the Helioseismic and Magnetic Imager ${ }^{42}$ daily updated synoptic maps of the radial magnetic field component on 26 January 2012 as the lower boundary. We further calculate the locations of all the null points and separators in the hemisphere containing the reconnection region. However, we cannot find any null points or separators in the source region of the reconnection event. It implies that the null point or separator responsible for the reconnection event is probably formed during the initial stages of the eruption. Note that, as the magnetic data were measured 1 day before the event, a possible evolution of the photospheric magnetic field could change this conclusion.

Uncertainty analysis. The errors of the DEM-weighted temperature and total EM depend on the errors of the DEM results, which come mainly from uncertainties in the temperature response functions of AIA including non-ionization equilibrium effects, non-thermal populations of electrons, modifications of dielectronic recombination rates, radiative transfer effects and even the unknown filling factor of the plasma ${ }^{43}$. Three representative DEM curves are shown in Supplementary Fig. 1b, from which one can find that the DEM solutions are well constrained in the temperature range $5.7 \leq \log T \leq 7.1$. To ensure the accuracy of the regularized inversion method, we also calculate the DEM with the forward fitting method ${ }^{44}$ and find that the two results are very similar.

A possible deviation in the $3 \mathrm{D}$ reconstruction of magnetic topology mainly comes from the uncertainty in identifying the same feature from two different perspectives. However, this does not affect qualitatively the global 3D topology. The uncertainty in displaying the heated region is mostly from the assumption that the filling factor is 1 and the $3 \mathrm{D}$ temperature distribution in the hot region is of cylindrical symmetry with the cross-section of the cylinder corresponding to the DEM-weighted 2D temperature map.

Code availability. The codes 'scc_measure.pro', 'aia_rfilter.pro', and 'sobel.pro' used in the above analysis are available at the website http://www.lmsal.com/ solarsoft/.

\section{References}

1. Priest, E. \& Forbes, T. Magnetic reconnection: MHD theory and applications (Cambridge Univ. Press, 2000).

2. Dai, Z. G., Wang, X. Y., Wu, X. F. \& Zhang, B. X-ray flares from postmerger millisecond pulsars. Science 311, 1127-1129 (2006).

3. Balbus, S. A. \& Hawley, J. F. Instability, turbulence, and enhanced transport in accretion disks. Rev. Mod. Phys. 70, 1-53 (1998).

4. Yuan, F., Lin, J., Wu, K. \& Ho, L. C. A magnetohydrodynamical mode for the formation of episodic jets. Mon. Not. R. Astron. Soc 395, 2183-2188 (2009).

5. Shibata, K. et al. Chromospheric anemone jets as evidence of ubiquitous reconnection. Science 318, 1591-1594 (2007).

6. Sturrock, P. A. Model of the high-energy phase of solar flares. Nature 211, 695-697 (1966).

7. Phan, T. D. et al. A magnetic reconnection X-line extending more than 390 Earth radii in the solar wind. Nature 439, 175-178 (2006).

8. Xiao, C. J. et al. Satellite observations of separator-line geometry of threedimensional magnetic reconnection. Nat. Phys. 3, 609-613 (2007).

9. Yamada, M., Kulsrud, R. \& Ji, H. Magnetic reconnection. Rev. Mod. Phys. 82, 603-664 (2010).

10. Zhong, J. et al. Modelling loop-top X-ray source and reconnection outflows in solar flares with intense lasers. Nat. Phys. 6, 984-987 (2010).

11. Priest, E. Magnetohydrodynamics of the Sun (Cambridge Univ. Press, 2014)

12. Paschmann, G. et al. Plasma acceleration at the earth's magnetopause-Evidence for reconnection. Nature 282, 243-246 (1979).

13. Masuda, S., Kosugi, T., Hara, H., Tsuneta, S. \& Ogawara, Y. A loop-top hard $\mathrm{X}$-ray source in a compact solar flare as evidence for magnetic reconnection. Nature 371, 495-497 (1994).

14. Li, L. \& Zhang, J. Observations of the magnetic reconnection signature of an M2 flare on 2000 March 23. Astrophys. J. 703, 877-882 (2009).

15. Yokoyama, T., Akita, K., Morimoto, T., Inoue, K. \& Newmark, J. Clear evidence of reconnection inflow of a solar flare. Astrophys. J. Lett. 546, L69-L72 (2001).

16. McKenzie, D. E. \& Savage, S. L. Quantitative examination of supra-arcade downflows in eruptive solar flares. Astrophys. J. 697, 1569-1577 (2009).

17. Sui, L. \& Holman, G. D. Evidence for the formation of a large-scale current sheet in a solar flare. Astrophys. J. Lett. 596, L251-L254 (2003).

18. Lin, J. et al. Direct observations of the magnetic reconnection site of an eruption on 2003 November 18. Astrophys. J. 622, 1251-1264 (2005). 
19. Su, Y. et al. Imaging coronal magnetic-field reconnection in a solar flare. Nat. Phys. 9, 489-493 (2013).

20. Yang, S., Zhang, J. \& Xiang, Y. Magnetic reconnection between small-scale loops observed with the new vacuum solar telescope. Astrophys. J. Lett. 798, L11 (2015).

21. Priest, E. R. \& Pontin, D. I. Three-dimensional null point reconnection regimes. Phys. Plasma 16, 122101 (2009)

22. Longcope, D. W., McKenzie, D. E., Cirtain, J. \& Scott, J. Observations of separator reconnection to an emerging active region. Astrophys. J. 630, 596-614 (2005).

23. Parnell, C. E., Haynes, A. L. \& Galsgaard, K. Structure of magnetic separators and separator reconnection. J. Geophys. Res. 115, A02102 (2010).

24. Aulanier, G., Pariat, E. \& Démoulin, P. Current sheet formation in quasiseparatrix layers and hyperbolic flux tubes. Astron. Astrophys. 444, 961-976 (2005).

25. Byrne, J. P., Maloney, S. A., McAteer, R. T. J., Refojo, J. M. \& Gallagher, P. T. Propagation of an Earth-directed coronal mass ejection in three dimensions. Nat. Commun. 1, 74 (2010).

26. Feng, L. et al. Morphological evolution of a three-dimensional coronal mass ejection cloud reconstructed from three viewpoints. Astrophys. J. 751, 18 (2012).

27. Howard, R. A. et al. Sun earth connection coronal and heliospheric investigation (SECCHI). Space Sci. Rev. 136, 67-115 (2008).

28. Lemen, J. R. et al. The atmospheric imaging assembly (AIA) on the solar dynamics observatory (SDO). Solar Phys. 275, 17-40 (2012).

29. Gibson, S. E. et al. Three-dimensional morphology of a coronal prominence cavity. Astrophys. J. 724, 1133-1146 (2010).

30. Cheng, X., Zhang, J., Liu, Y. \& Ding, M. D. Observing flux rope formation during the impulsive phase of a solar eruption. Astrophys. J. Lett. 732, L25 (2011).

31. Zhang, J., Cheng, X. \& Ding, M. D. Observation of an evolving magnetic flux rope before and during a solar eruption. Nat. Commun. 3, 747 (2012).

32. Brueckner, G. E. et al. The large angle spectroscopic coronagraph (LASCO). Solar Phys. 162, 357-402 (1995)

33. Priest, E. R. \& Forbes, T. G. The magnetic nature of solar flares. Astron. Astrophys. Rev. 10, 313-377 (2002).

34. Sakurai, T. Computational modeling of magnetic fields in solar active regions. Space Sci. Rev. 51, 11-48 (1989).

35. Forbes, T. G. \& Acton, L. W. Reconnection and field line shrinkage in solar flares. Astrophys. J. 459, 330-341 (1996).

36. Gopalswamy, N. Properties of interplanetary coronal mass ejections. Space Sci. Rev. 124, 145-168 (2006)

37. Cheng, X. et al. Formation of a double-decker magnetic flux rope in the sigmoidal solar active region 11520. Astrophys. J. 789, 93 (2014).

38. Pontin, D. I. Theory of magnetic reconnection in solar and astrophysical plasmas. Phil. Trans. R. Soc. A 370, 3169-3192 (2012).

39. Shibata, K. et al. Hot-plasma ejections associated with compact-loop solar flares. Astrophys. J. Lett. 451, L83-L85 (1995).

40. Kliem, B. \& Török, T. Torus instability. Phys. Rev. Lett. 96, 255002 (2006)
41. Hannah, I. G. \& Kontar, E. P. Differential emission measures from the regularized inversion of Hinode and SDO data. Astron. Astrophys. 539, A146 (2012).

42. Schou, J. et al. Design and ground calibration of the helioseismic and magnetic imager (HMI) instrument on the Solar Dynamics Observatory (SDO). Solar Phys. 275, 229-259 (2012).

43. Judge, P. G. Coronal emission lines as thermometers. Astrophys. J. 708, 1238-1240 (2010).

44. Cheng, X., Zhang, J., Saar, S. H. \& Ding, M. D. Differential emission measure analysis of multiple structural components of coronal mass ejections in the inner corona. Astrophys. J. 761, 62 (2012).

\section{Acknowledgements}

SDO is a mission of NASA's Living With a Star Program, STEREO is the third mission in NASA's Solar Terrestrial Probes Program, and SOHO is a mission of international cooperation between ESA and NASA. X.C., J.Q.S., M.D.D., Y.G., P.F.C. and C.F. are supported by NSFC through grants 11303016, 11373023, 11203014 and 11025314, and by NKBRSF through grants $2011 \mathrm{CB} 811402$ and 2014CB744203. C.E.P. and S.J.E. are supported by the UK STFC. J.Z. is supported by US NSF AGS-1249270 and AGS-1156120

\section{Author contributions}

X.C. and J.Q.S. analysed the observational data and contributed equally to the work. X.C initiated the study. M.D.D. supervised the project and led the discussions. Y.G. joined part of the data analysis. C.E.P. and S.J.E. performed the magnetic field extrapolation. X.C. wrote the first draft. M.D.D. and E.R.P. made major revisions of the manuscript. The remaining authors discussed the results and commented on the manuscript.

\section{Additional information}

Supplementary Information accompanies this paper at http://www.nature.com/ naturecommunications

Competing financial interests: The authors declare no competing financial interests.

Reprints and permission information is available online at http://npg.nature.com/ reprintsandpermissions/

How to cite this article: Sun, J. Q. et al. Extreme ultraviolet imaging of three-dimensional magnetic reconnection in a solar eruption. Nat. Commun. 6:7598 doi: $10.1038 /$ ncomms8598 (2015).

cc (i) This work is licensed under a Creative Commons Attribution 4.0 International License. The images or other third party material in this article are included in the article's Creative Commons license, unless indicated otherwise in the credit line; if the material is not included under the Creative Commons license, users will need to obtain permission from the license holder to reproduce the material. To view a copy of this license, visit http://creativecommons.org/licenses/by/4.0/ 